\title{
Existence and Stability of the Solution of a Nonlinear Boundary Value Problem
}

\author{
Agneta M. Balint ${ }^{1}$ and Stefan Balint ${ }^{2}$ \\ ${ }^{1}$ Faculty of Physics, West University of Timisoara, Bulevard V.Parvan 4, 300223 Timisoara, Romania \\ ${ }^{2}$ Faculty of Mathematics and Computer Science, West University of Timisoara, Bulevard V.Parvan 4, \\ 300223 Timisoara, Romania
}

Correspondence should be addressed to Agneta M. Balint, balint@physics.uvt.ro

Received 31 July 2012; Accepted 5 November 2012

Academic Editor: Carlos Lizama

Copyright ( 2012 A. M. Balint and S. Balint. This is an open access article distributed under the Creative Commons Attribution License, which permits unrestricted use, distribution, and reproduction in any medium, provided the original work is properly cited.

The purpose is to find conditions assuring the existence of solutions for a nonlinear, boundary value problem in case of the axis-symmetric Young-Laplace differential equation. The equation describes the capillary surface between two static fluids. Necessary or sufficient conditions are found for the existence of a solution. The static stability of the obtained solution is also analyzed and stability or instability results are revealed. For the NdYAG microfiber growth, by the pullingdown method, numerical illustrations are given.

\section{Motivation of the Mathematical Considerations}

The near one-dimensional single crystal fibers have attracted some attention on the applications of optical and electronic devices [1-3]. There are several methods for growing fiber crystals [4], such as the edge-defined film-fed growth (EFG) and floating zone (pedestal growth) methods. The micro-pulling-down ( $\mu$-PD) process, a variant of the inverse EFG, developed by Fukuda's laboratory in Japan [5-9], has been shown promising in producing single crystal fibers with good diameter control and concentration uniformity. Several oxide [5-7] and semiconductor fibers $[8,9]$ have been grown. The grown diameter ranges are 10$1500 \mu \mathrm{m}$ for oxides and about $300-900 \mu \mathrm{m}$ for semiconductors. According to [10], for this process some simple theoretical analyses for the operation limit [11] and solute distribution $[12,13]$ have been performed, and no detailed modeling has been conducted. In the previous reports the melt convection was ignored, and heat and mass transfer coupled with the capillary shaping and solidification have not been analyzed. 
Since the system is small, the measurements of flow, dopant, and temperature distributions are difficult. In [10] a detailed numerical simulation is conducted in a selfconsistent manner to analyze the $\mu$-PD process for $\mathrm{Ge}_{x} \mathrm{Si}_{1-x}$ fibers; $x=0.05$. The system of partial differential equations describing the melt flow, heat, and mass transfer, the crystallization front and meniscus, and the grown fiber diameter are solved numerically in stationary case for various process parameters. The model formulation and analysis are very similar to those developed in $[14,15]$ and appropriate for the analysis of the radial segregation. Since the model presented in [10] is a stationary model, it cannot be used for the analysis of the axial segregation, which can be relevant in $\mu$-PD process, as is reported in [16].

In this paper the meniscus shape and size, appearing in $\mu$-PD, are evaluated in function of a parameter $p$, which incorporates the hydrostatic pressure of the melt column situated behind the meniscus, the hydrodynamic pressure associated to the thermoconvection in the meniscus, the Marangoni pressure associated to the Marangoni convection, and the gas pressure in front of the free surface of the meniscus. The obtained results can be useful for defining the geometry in case of the simulation of the growth process.

\section{Formulation of the Mathematical Problem}

For single crystal fiber growth by $\mu$-PD method, the free surface of the meniscus at each moment of time is described by the Young-Laplace equation [17, 18]:

$$
r \cdot\left(\frac{1}{R_{1}}+\frac{1}{R_{2}}\right)=P_{a}-P_{b}
$$

Here $\gamma$ is the melt surface tension; $1 / R_{1}, 1 / R_{2}$ denote the main normal curvatures of the free surface at a point $M$ of the free surface; $P_{a}$ is the pressure in front of the free surface; $P_{b}$ is the pressure behind the free surface (Figure 1).

The pressure $P_{a}$ in front of the free surface is equal to the pressure of the gas introduced in the furnace and thereafter is denoted by $p_{g}\left(P_{a}=p_{g}\right)$.

The pressure $P_{b}$ behind the free surface is the sum of the hydrodynamic pressure $p_{m}$ in the meniscus melt (due to the thermal convection), the Marangoni pressure $p_{M}$ due to the Marangoni convection, and the hydrostatic pressure of the melt column behind the free surface equal to $\rho \cdot g \cdot\left(z+h_{m}\right)$ (see Figure 1). Here $\rho$ denotes the melt density; $g$ is the gravity acceleration; $z$ is the coordinate of $M$ with respect to the $O z$ axis, directed vertically downwards; $h_{m}$ denotes the melt column height between the horizontal crucible melt level and the shaper bottom level (see Figure 1).

The pressure difference $P_{a}-P_{b}$ across the free surface is denoted usually by $\Delta p$ and, according to the above explanations,

$$
\Delta p=p_{g}-p_{m}-p_{M}-\rho \cdot g \cdot\left(z+h_{m}\right)
$$

The part $p$ given by:

$$
p=-p_{g}+\rho \cdot g \cdot h_{m}+p_{m}+p_{M}
$$

of the pressure difference $\Delta p$ is considered to be known. That is because the major part $-p_{g}+$ $\rho \cdot g \cdot h_{m}$ of $p$ is known, and $p_{m}+p_{M}$ in general is small with respect to $-p_{g}+\rho \cdot g \cdot h_{m}$ and is 

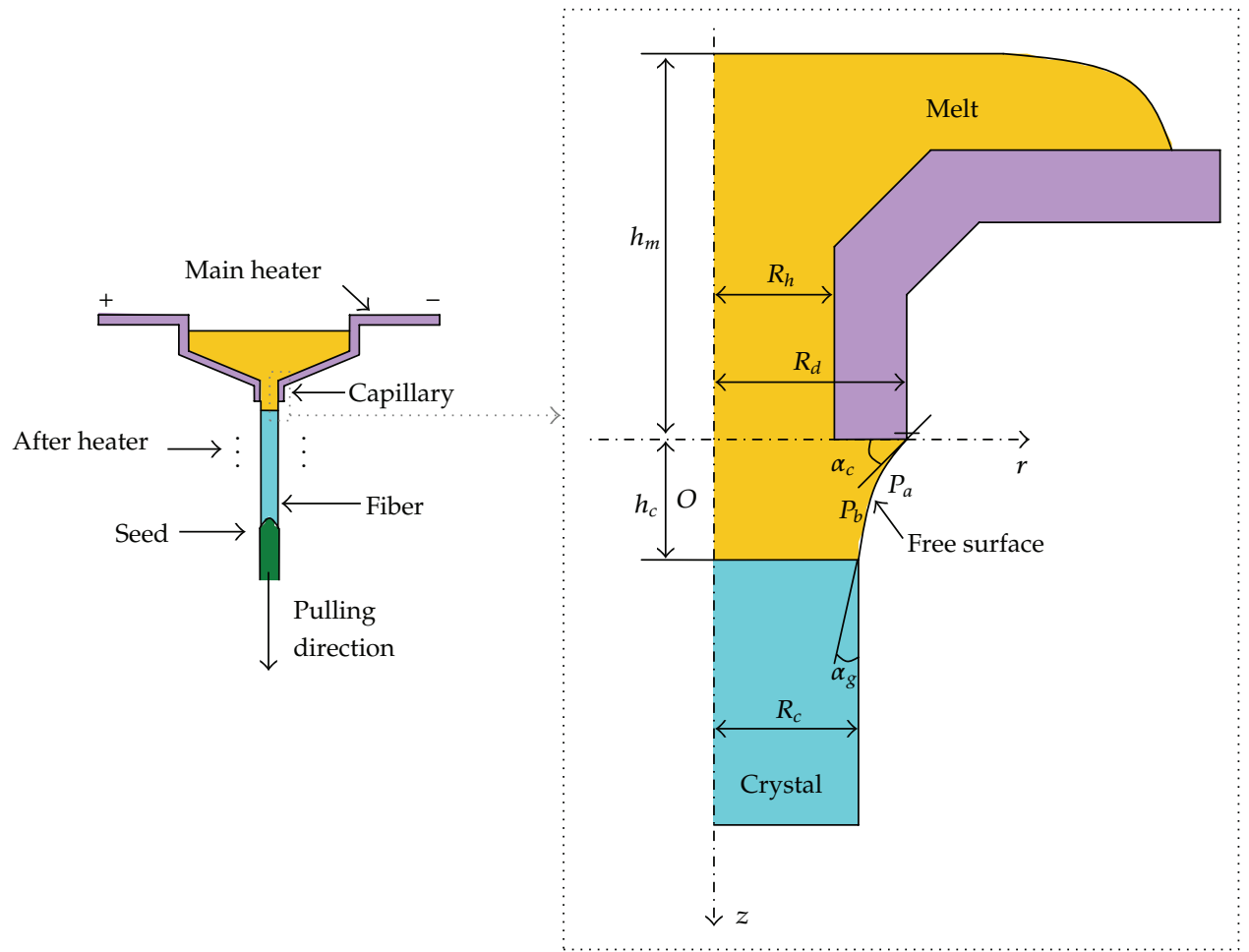

Figure 1: Schematic diagram of the $\mu$-pulling-down fiber growth process.

mainly determined by the thermal conditions. What is important to retain is that $p$ does not depend on spatial coordinate, but it can depend on the moment of time $t$.

In the above terms the Young-Laplace equation (2.1) can be written as

$$
r \cdot\left(\frac{1}{R_{1}}+\frac{1}{R_{2}}\right)=-\rho \cdot g \cdot z-p
$$

To calculate the meniscus-free surface shape and size are convenient to employ the YoungLaplace equation (2.4) in its differential form. This form can be obtained also as a necessary condition for the minimum of the free energy of the melt column $[17,18]$. surface is

The differential equation of the meridian curve for axis-symmetric meniscus-free

$$
z^{\prime \prime}=-\frac{\rho \cdot g \cdot z+p}{\gamma}\left[1+\left(z^{\prime}\right)^{2}\right]^{3 / 2}-\frac{1}{r} \cdot\left[1+\left(z^{\prime}\right)^{2}\right] \cdot z^{\prime} \quad \text { for } R_{c} \leq r \leq R_{d}=\text { shaper radius }
$$


If the solution $z(r)$ of (2.5) is the meridian curve of the free surface of a meniscus on the interval $\left[R_{c}, R_{d}\right]$, then it verifies the following boundary conditions:

$$
\begin{gathered}
z^{\prime}\left(R_{c}\right)=-\tan \left(\frac{\pi}{2}-\alpha_{g}\right) ; \quad z\left(R_{c}\right)=h_{c}>0, \\
z^{\prime}\left(R_{d}\right)=-\tan \alpha_{c} ; \quad z\left(R_{d}\right)=0, \\
z(r) \text { is strictly decreasing on }\left[R_{c}, R_{d}\right] .
\end{gathered}
$$

The first condition in (2.6) expresses that at the three-phase point $\left(R_{c}, h_{c}\right)$, where the thermal conditions for solidification have to be realized, the angle between the tangent line to the meridian curve of the free surface and the vertical is equal to the growth angle. If this condition is satisfied during the whole process, then the tangent to the fiber surface at the three-phase point is vertical; that is, the fiber diameter is constant.

The second condition in (2.6) expresses that the coordinate of the crystallization front is equal to $h_{c}>0$.

The first condition in (2.7) expresses that, at the three-phase point $\left(R_{d}, 0\right)$, where the meridian curve touches the outer edge of the shaper, the catching angle is equal to $\alpha_{c}$.

The second condition in (2.7) expresses that at the three-phase point $\left(R_{d}, 0\right)$ the meridian curve is fixed to the outer edge of the shaper.

Condition (2.8) expresses the fact that the meniscus shape is relatively simple.

The determination of $z(r)$ for which (2.5) and (2.6) hold is a nonlinear boundary value problem (NLBVP).

Equation (2.5) is the Euler equation of the free energy functional of the melt column:

$$
\begin{gathered}
I(z)=\int_{R_{c}}^{R_{d}}\left\{r \cdot\left[1+\left(z^{\prime}\right)^{2}\right]^{1 / 2}-\frac{1}{2} \cdot \rho \cdot g \cdot z^{2}-p \cdot z\right\} \cdot r \cdot d r, \\
z\left(R_{c}\right)=h_{c}>0, \quad z\left(R_{d}\right)=0 .
\end{gathered}
$$

The nonlinear boundary value problem (NLBVP), (2.5) and (2.6), is equivalent to the NLBVP:

$$
\begin{aligned}
\frac{d z}{d r} & =-\tan \alpha, \\
\frac{d \alpha}{d r} & =\frac{\rho \cdot g \cdot z+p}{r} \cdot \frac{1}{\cos \alpha}-\frac{1}{r} \cdot \tan \alpha, \\
z\left(R_{c}\right) & =h_{c}, \quad \alpha\left(R_{c}\right)=\frac{\pi}{2}-\alpha_{g}, \\
z\left(R_{d}\right) & =0, \quad \alpha\left(R_{d}\right)=\alpha_{c} .
\end{aligned}
$$

\section{Existence of the Solution}

Consider the differential equation:

$$
z^{\prime \prime}=-\frac{\rho \cdot g \cdot z+p}{r}\left[1+\left(z^{\prime}\right)^{2}\right]^{3 / 2}-\frac{1}{r} \cdot\left[1+\left(z^{\prime}\right)^{2}\right] \cdot z^{\prime} \quad \text { for } 0<R_{c} \leq r \leq R_{d} R_{d}>0 .
$$

And $\alpha_{c}, \alpha_{g}$ such that $\alpha_{c}, \alpha_{g} \in(0, \pi / 2)$. 
Definition 3.1. A solution $z=z(r)$ of (3.1) is a globally convex solution of the NLBVP (2.5) and (2.6) on the interval $\left[R_{c}, R_{d}\right]\left(0<R_{c}<R_{d}\right)$ if it possesses the following properties:

(a) $z^{\prime}\left(R_{c}\right)=-\tan \left(\pi / 2-\alpha_{g}\right)$,

(b) $z^{\prime}\left(R_{d}\right)=-\tan \alpha_{c}$

(c) $z\left(R_{d}\right)=0$ and $z(r)$ is strictly decreasing on $\left[R_{c}, R_{d}\right]$,

(d) $z^{\prime \prime}(r)>0$ for all $r \in\left[R_{c}, R_{d}\right]$,

A solution $z(r)$ of (3.1) is a globally-concave solution of the NLBVP (2.5), (2.6) on the interval $\left[R_{c}, R_{d}\right]\left(0<R_{c}<R_{d}\right)$ if verifies (a) (b) (c) and in addition the following inequality holds:

(e) $z^{\prime \prime}(r)<0$ for all $r \in\left[R_{c}, R_{d}\right]$.

Remark 3.2. If $\alpha_{c}<\pi / 2-\alpha_{g}$, then for a globally concave solution $z(r)\left(z^{\prime \prime}(r)<0\right)$ of (3.1), which satisfies (b) and (c), there is no $R_{c} \in\left(0, R_{d}\right)$ such that (a) holds. Therefore, if $\alpha_{c}<$ $\pi / 2-\alpha_{g}$ and we are interested in solutions of (3.1) which satisfy (b) and (c) and are not globally concave.

If $\pi / 2-\alpha_{g}<\alpha_{c}$, then for a globally convex solution $z(r)\left(z^{\prime \prime}(r)<0\right)$ of (3.1), which satisfies (b) and (c), and there is no $R_{c} \in\left(0, R_{d}\right)$ such that a. holds. Therefore, if $\pi / 2-\alpha_{g}<\alpha_{c}$, we are interested in solutions of (3.1) which satisfy (b) and (c) and are not globally convex.

Theorem 3.3 (necessary condition for the existence of a globally convex solution). If $\alpha_{c}<$ $\pi / 2-\alpha_{g}$ and there exists a globally convex solution $z(r)$ of the NLBVP (2.5) and (2.6), on $\left[R_{c}, R_{d}\right]$, then the following inequalities hold:

$$
\begin{gathered}
\gamma \cdot \frac{\alpha_{c}+\alpha_{g}-\pi / 2}{R_{d}-R_{c}} \cdot \cos \alpha_{c}-\rho \cdot g \cdot\left[R_{d}-R_{c}\right] \cdot \tan \left(\frac{\pi}{2}-\alpha_{g}\right)+\frac{\gamma}{R_{d}} \cdot \sin \alpha_{c} \\
\leq p \leq \gamma \cdot \frac{\alpha_{c}+\alpha_{g}-\pi / 2}{R_{d}-R_{c}} \cdot \sin \alpha_{g}+\frac{\gamma}{R_{c}} \cdot \cos \alpha_{g} .
\end{gathered}
$$

Proof. Let $z(r)$ be a globally convex solution of the problem (2.5) and (2.6) on $\left[R_{c}, R_{d}\right]$ and $\alpha(r)=-\arctan z^{\prime}(r)$. The function $\alpha(r)$ verifies the following quation:

$$
\alpha^{\prime}(r)=\frac{\rho \cdot g \cdot z(r)+p}{r} \cdot \frac{1}{\cos \alpha(r)}-\frac{1}{r} \cdot \tan \alpha(r)
$$

and the boundary conditions:

$$
\alpha\left(R_{c}\right)=\frac{\pi}{2}-\alpha_{g}, \quad \alpha\left(R_{d}\right)=\alpha_{c} .
$$

Hence, according to the Lagrange mean value theorem, there exists $r^{\prime} \in\left(R_{c}, R_{d}\right)$ such that the following equality holds:

$$
p=\gamma \cdot \frac{\alpha_{c}+\alpha_{g}-\pi / 2}{R_{d}-R_{c}} \cdot \cos \alpha\left(r^{\prime}\right)-\rho \cdot g \cdot z\left(r^{\prime}\right)+\frac{\gamma}{r^{\prime}} \cdot \sin \alpha\left(r^{\prime}\right) .
$$


Since $z^{\prime \prime}(r)>0$ on $\left[R_{c}, R_{d}\right], z^{\prime}(r)$ is strictly increasing, and $\alpha(r)=-\arctan z^{\prime}(r)$ is strictly decreasing on $\left[R_{c}, R_{d}\right]$. Therefore, the following inequalities hold:

$$
\begin{gathered}
\alpha_{c} \leq \alpha\left(r^{\prime}\right) \leq \frac{\pi}{2}-\alpha_{g}, \\
\sin \alpha_{g} \leq \cos \alpha\left(r^{\prime}\right) \leq \cos \alpha_{c}, \\
\sin \alpha_{c} \leq \sin \alpha\left(r^{\prime}\right) \leq \cos \alpha_{g}, \\
-\rho \cdot g \cdot\left(R_{d}-R_{c}\right) \cdot \tan \left(\frac{\pi}{2}-\alpha_{g}\right) \leq-\rho \cdot g \cdot z\left(r^{\prime}\right) \leq-\rho \cdot g \cdot\left(R_{d}-r^{\prime}\right) \cdot \tan \alpha_{c} .
\end{gathered}
$$

Equality (3.5) and inequalities (3.6) imply (3.2).

Corollary 3.4. In terms of the ratio $n=\left(R_{d} / R_{c}\right)(n>1)$ the inequalities (3.2) can be written as

$$
\begin{gathered}
r \cdot \frac{\alpha_{c}+\alpha_{g}-\pi / 2}{R_{d}} \cdot \frac{n}{n-1} \cdot \cos \alpha_{c}-\rho \cdot g \cdot R_{d} \cdot \frac{(n-1)}{n} \cdot \tan \left(\frac{\pi}{2}-\alpha_{g}\right)+\frac{\gamma}{R_{d}} \cdot \sin \alpha_{c} \\
\leq p \leq \gamma \cdot \frac{\alpha_{c}+\alpha_{g}-\pi / 2}{R_{d}} \cdot \frac{n}{n-1} \cdot \sin \alpha_{g}+\frac{\gamma}{R_{d}} \cdot n \cdot \cos \alpha_{g} .
\end{gathered}
$$

Corollary 3.5. If $n \rightarrow+\infty$, then $R_{c} \rightarrow 0$ and (3.7) become

$$
\gamma \cdot \frac{\alpha_{c}+\alpha_{g}-\pi / 2}{R_{d}} \cdot \cos \alpha_{c}-\rho \cdot g \cdot R_{d} \cdot \tan \left(\frac{\pi}{2}-\alpha_{g}\right)+\frac{\gamma}{R_{d}} \cdot \sin \alpha_{c} \leq p<+\infty
$$

If $n \rightarrow 1$, then $R_{c} \rightarrow R_{d}$ and $p \rightarrow-\infty$.

Theorem 3.6 (necessary condition for the existence of a globally concave solution). If $\pi / 2-$ $\alpha_{g}<\alpha_{c}$, and there exists a globally concave solution $z(r)\left(z^{\prime \prime}(r)<0\right)$ of the NLBVP $(2.5)$ and $(2.6)$ on $\left[R_{c}, R_{d}\right]$, then the following inequalities hold:

$$
\begin{gathered}
\gamma \cdot \frac{\alpha_{c}+\alpha_{g}-\pi / 2}{R_{d}-R_{c}} \cdot \cos \alpha_{c}-\rho \cdot g \cdot\left[R_{d}-R_{c}\right] \cdot \tan \alpha_{c}+\frac{\gamma}{R_{d}} \cdot \cos \alpha_{g} \\
\leq p \leq \gamma \cdot \frac{\alpha_{c}+\alpha_{g}-\pi / 2}{R_{d}-R_{c}} \cdot \sin \alpha_{g}+\frac{\gamma}{R_{c}} \cdot \sin \alpha_{c} .
\end{gathered}
$$


Proof. Since $z^{\prime \prime}(r)<0$ on $\left[R_{c}, R_{d}\right], z^{\prime}(r)$ is strictly decreasing and $\alpha(r)=-\arctan z^{\prime}(r)$ is strictly increasing on $\left[R_{c}, R_{d}\right]$. Therefore the following inequalities hold:

$$
\begin{gathered}
\frac{\pi}{2}-\alpha_{g} \leq \alpha(r) \leq \alpha_{c}, \\
\cos \alpha_{c} \leq \cos \alpha(r) \leq \sin \alpha_{g},
\end{gathered}
$$

$\cos \alpha_{g} \leq \sin \alpha(r) \leq \sin \alpha_{c}$

$$
-\rho \cdot g \cdot\left(R_{d}-R_{c}\right) \cdot \tan \alpha_{c} \leq-\rho \cdot g \cdot z(r) \leq-\rho \cdot g \cdot\left(R_{d}-r^{\prime}\right) \cdot \tan \left(\frac{\pi}{2}-\alpha_{g}\right)
$$

for every $r \in\left[R_{c}, R_{d}\right]$.

Equality (3.5) and inequalities (3.10) imply inequalities (3.9).

Corollary 3.7. In terms of the ratio $n=\left(R_{d} / R_{c}\right)(n>1)$ the inequalities (3.9) can be written as

$$
\begin{gathered}
\gamma \cdot \frac{\alpha_{c}+\alpha_{g}-\pi / 2}{R_{d}} \cdot \frac{n}{n-1} \cdot \cos \alpha_{c}-\rho \cdot g \cdot R_{d} \cdot \frac{(n-1)}{n} \cdot \tan \alpha_{c}+\frac{\gamma}{R_{d}} \cdot \cos \alpha_{g} \\
\leq p \leq \gamma \cdot \frac{\alpha_{c}+\alpha_{g}-\pi / 2}{R_{d}} \cdot \frac{n}{n-1} \cdot \sin \alpha_{g}+\frac{\gamma}{R_{d}} \cdot n \cdot \sin \alpha_{c} .
\end{gathered}
$$

Corollary 3.8. If $n \rightarrow+\infty$, then $R_{c} \rightarrow 0$ and (3.11) become

$$
\gamma \cdot \frac{\alpha_{c}+\alpha_{g}-\pi / 2}{R_{d}} \cdot \cos \alpha_{c}-\rho \cdot g \cdot R_{d} \cdot \tan \alpha_{c}+\frac{\gamma}{R_{d}} \cdot \cos \alpha_{g} \leq p<+\infty
$$

If $n \rightarrow 1$, then $R_{c} \rightarrow R_{d}$ and $p \rightarrow+\infty$.

Theorem 3.9 (necessary condition for the existence of a convex-concave solution). If for $\alpha_{c}<$ $\pi / 2-\alpha_{g}$ and $p>\left(\gamma / R_{d}\right) \cdot \sin \alpha_{c}$ and there exists a solution $z(r)$ of the NLBVP (2.5) and (2.6) on the interval $\left[R_{c}, R_{d}\right]\left(0<R_{c}<R_{d}\right)$ and then for $p$ the following inequalities hold:

$$
\frac{\gamma}{R_{d}} \cdot \sin \alpha_{c}<p<\frac{\gamma}{R_{c}} \cdot \cos \alpha_{g}
$$

Proof. Since $p>\left(\gamma / R_{d}\right) \cdot \sin \alpha_{c}$, we have $\left(p / \gamma-1 / R_{d}\right) \cdot \sin \alpha_{c}>0$ and hence $\alpha^{\prime}\left(R_{d}\right)=\left(1 / \cos \alpha_{c}\right)$. $\left[\left(p / \gamma-1 / R_{d}\right) \cdot \sin \alpha_{c}\right]>0$. It follows that, for $r<R_{d}, r$ close to $R_{d}$, the following inequality: $\alpha(r)<\alpha\left(R_{d}\right)=\alpha_{c}$ holds. Since $\alpha\left(R_{c}\right)=\pi / 2-\alpha_{g}>\alpha_{c}$, it follows that there exists $r^{\prime} \in\left(R_{c}, R_{d}\right)$ such that $\alpha^{\prime}\left(r^{\prime}\right)=0\left(r^{\prime}\right.$ is the point where $\alpha(r)$ is minimum). The equality $\alpha^{\prime}\left(r^{\prime}\right)=0$ implies that the following equality holds:

$$
p=\frac{\gamma}{r^{\prime}} \cdot \sin \alpha\left(r^{\prime}\right)-\rho \cdot g \cdot z\left(r^{\prime}\right)
$$

Taking into account the fact that $z(r)$ is a decreasing function on $\left[R_{c}, R_{d}\right]$, we deduce that $p<\left(\gamma / R_{c}\right) \cdot \cos \alpha_{g}$. 
Theorem 3.10 (necessary condition for the existence of a concave-convex solution). If for $\alpha_{c}>$ $\pi / 2-\alpha_{g}$ and $p<\left(\gamma / R_{d}\right) \cdot \sin \alpha_{c}$, there exists a solution $z(r)$ of the NLBVP (2.5) and (2.6) on the interval $\left[R_{c}, R_{d}\right]\left(0<R_{c}<R_{d}\right)$, then for $p$ the following inequalities hold:

$$
\frac{\gamma}{R_{d}} \cdot \cos \alpha_{g}<p<\frac{\gamma}{R_{d}} \cdot \sin \alpha_{c} .
$$

Proof. Since $p<\left(\gamma / R_{d}\right) \cdot \sin \alpha_{c}$, we have $(p / \gamma)-1 / R_{d} \cdot \sin \alpha_{c}<0$ and hence $\alpha^{\prime}\left(R_{d}\right)=\left(1 / \cos \alpha_{c}\right)$. $\left[\left(p / \gamma-1 / R_{d}\right) \cdot \sin \alpha_{c}\right]<0$. It follows that, for $r<R_{d}, r$ close to $R_{d}$, the inequality $\alpha_{c}=$ $\alpha\left(R_{d}\right)<\alpha(r)$ holds. Since $\alpha\left(R_{c}\right)=\pi / 2-\alpha_{g}<\alpha_{c}$, it follows that there exists $r^{\prime} \in\left(R_{c}, R_{d}\right)$ such that $\alpha^{\prime}\left(r^{\prime}\right)=0\left(r^{\prime}\right.$ is the point where $\alpha(r)$ is maximum). The equality $\alpha^{\prime}\left(r^{\prime}\right)=0$ implies that the equality (3.14) holds. Taking into account the fact that $z(r)$ is a decreasing function on $\left[R_{c}, R_{d}\right]$, we deduce (3.15).

Theorem 3.11. If for $\alpha_{c}<\pi / 2-\alpha_{g}, p<\left(\gamma / R_{d}\right) \cdot \sin \alpha_{c}$ there exists a solution $z(r)$ of the NLBVP (2.5) and (2.6), on the interval $\left[R_{c}, R_{d}\right]\left(0<R_{c}<R_{d}\right)$ and $z^{\prime \prime}\left(R_{c}\right)<0$, then $z(r)$ is concave convex on $\left[R_{c}, R_{d}\right]$ and there exists $R_{c}^{1}, R_{c}<R_{c}^{1}<R_{d}$ such that $z(r)$ is a globally convex solution of the $\operatorname{NLBVP}(2.5)$ and (2.6) on $\left[R_{c}^{1}, R_{d}\right]$. Moreover for $p$ the inequality (3.15) holds.

Proof. Since $p<\left(\gamma / R_{d}\right) \cdot \sin \alpha_{c}$, we have $\alpha^{\prime}\left(R_{d}\right)<0$. Inequality $z^{\prime \prime}\left(R_{c}\right)<0$ implies that $\alpha^{\prime}\left(R_{c}\right)>$ 0 . Hence, there exists $r^{\prime} \in\left(R_{c}, R_{d}\right)$ such that $\alpha^{\prime}\left(r^{\prime}\right)=0$. This equality implies that (3.14) holds. Taking into account the fact that $z(r)$ is a decreasing function on $\left[R_{c}, R_{d}\right]$, we deduce (3.15).

The existence of $R_{c}^{1}$ follows from the fact that $\pi / 2-\alpha_{g}=\alpha\left(R_{c}\right)<\alpha(r)$ for $r>R_{c}, r$ close to $R_{c}$ and $\alpha\left(R_{d}\right)=\alpha_{c}<\pi / 2-\alpha_{g}$.

Theorem 3.12. If for $\alpha_{c}>\pi / 2-\alpha_{g}, p>\left(\gamma / R_{d}\right) \cdot \sin \alpha_{c}$, there exists a solution $z(r)$ of the NLBVP (2.5) and (2.6) on the interval $\left[R_{c}, R_{d}\right]\left(0<R_{c}<R_{d}\right)$ and $z^{\prime \prime}\left(R_{c}\right)>0$, then $z(r)$ is a convex concave solution of the NLBVP (2.5) and (2.6) on $\left[R_{c}, R_{d}\right]$ and there exists $R_{c}^{1}, R_{c}<R_{c}^{1}<R_{d}$, such that $z(r)$ is a globally-concave solution of the NLBVP $(2.5),(2.6)$ on $\left[R_{c}^{1}, R_{d}\right]$. Moreover for $p$ the inequality (3.13) holds.

Proof. Since $p>\left(\gamma / R_{d}\right) \cdot \sin \alpha_{c}$, we have $\alpha^{\prime}\left(R_{d}\right)>0$. Inequality $z^{\prime \prime}\left(R_{c}\right)>0$ implies that $\alpha^{\prime}\left(r^{\prime}\right)=$ 0 . This equality implies that (3.14) holds. Taking into account the fact that $z(r)$ is a decreasing function on $\left[R_{c}, R_{d}\right]$, we deduce that (3.13) holds.

The existence of $R_{c}^{1}$ follows from the fact that $\pi / 2-\alpha_{g}=\alpha\left(R_{c}\right)>\alpha(r)$ for $r>R_{c}, r$ close to $R_{c}$ and $\alpha\left(R_{d}\right)=\alpha_{c}>\pi / 2-\alpha_{g}$.

Theorem 3.13 (sufficient condition for the existence of a globally convex solution). Let $n>1$ be a real number. If $\alpha_{c}<\pi / 2-\alpha_{g}$ and $p$ satisfies

$$
p<\gamma \cdot \frac{\alpha_{c}+\alpha_{g}-\pi / 2}{R_{d}} \cdot \frac{n}{n-1} \cdot \cos \alpha_{c}-\rho \cdot g \cdot R_{d} \cdot \frac{n-1}{n} \cdot \tan \left(\frac{\pi}{2}-\alpha_{g}\right)+\frac{\gamma}{R_{d}} \cdot \sin \alpha_{c}
$$

then there exists $R_{c}$ in the closed interval $\left[R_{d} / n, R_{d}\right]$ such that the solution of the initial value problem

$$
\begin{gathered}
z^{\prime \prime}=-\frac{\rho \cdot g \cdot z+p}{r}\left[1+\left(z^{\prime}\right)^{2}\right]^{3 / 2}-\frac{1}{r}\left[1+\left(z^{\prime}\right)^{2}\right] \cdot z^{\prime} \quad 0<r<R_{d}, R_{d}>0 \\
z\left(R_{d}\right)=0, \quad z^{\prime}\left(R_{d}\right)=-\tan \alpha_{c} \quad 0<\alpha_{c}<\frac{\pi}{2}-\alpha_{g}, \quad \alpha_{g} \in\left(0, \frac{\pi}{2}\right)
\end{gathered}
$$

is a globally convex solution of the NLBVP (2.5) and (2.6) on $\left[R_{c}, R_{d}\right]$. 
Proof. Consider the solution $z(r)$ of the initial value problem (3.17). Denote by $I$ the maximal interval on which the function $z(r)$ exists and by $\alpha(r)$ the function $\alpha(r)=-\arctan z^{\prime}(r)$ defined on $I$. for the function $\alpha(r)$ the following equality holds:

$$
\alpha^{\prime}(r)=\frac{1}{\cos \alpha}\left[\frac{p+\rho \cdot g \cdot z}{\gamma}-\frac{\sin \alpha}{r}\right] .
$$

Since

$$
\begin{gathered}
z^{\prime \prime}\left(R_{d}\right)=-\frac{\alpha^{\prime}\left(R_{d}\right)}{\cos ^{2} \alpha\left(R_{d}\right)}=-\frac{1}{\cos ^{3} \alpha\left(R_{d}\right)}\left[\frac{p}{\gamma}-\frac{\sin \alpha\left(R_{d}\right)}{R_{d}}\right]>0, \\
z^{\prime}\left(R_{d}\right)=-\tan \alpha_{c}<0 \\
z^{\prime}\left(R_{d}\right)>-\tan \left(\frac{\pi}{2}-\alpha_{g}\right)
\end{gathered}
$$

there exists $r^{\prime} \in I, 0<r^{\prime}<R_{d}$ such that for any $r \in\left[r^{\prime}, R_{d}\right]$ the following inequalities hold:

$$
z^{\prime \prime}(r)>0, \quad z^{\prime}(r) \leq-\tan \alpha_{c}<0, \quad z^{\prime}(r)>-\tan \left(\frac{\pi}{2}-\alpha_{g}\right) .
$$

Let now be $r_{*}$ defined by

$$
r_{*}=\inf \left\{r^{\prime} \in I \mid 0<r^{\prime}<R_{d} \text { such that for any } r \in\left[r^{\prime}, R_{d}\right] \text { inequalities (3.20) hold }\right\} .
$$

It is clear that $r_{*} \geq 0$ and for any $r \in\left(r_{*}, R_{d}\right]$ (3.20) hold. Moreover, $z^{\prime}\left(r_{*}+0\right)=\lim _{r \rightarrow r_{*}, r>r_{*}} z^{\prime}(r)$ exists and satisfies $z^{\prime}\left(r_{*}+0\right)<-\tan \alpha_{c}<0$ and $z^{\prime}\left(r_{*}+0\right) \geq-\tan \left(\pi / 2-\alpha_{g}\right)$. Hence $z\left(r_{*}+0\right)=$ $\lim _{r \rightarrow r_{*}, r>r_{*}} z(r)$ is finite, it is strictly positive and for every $r \in\left(r_{*}, R_{d}\right]$ the inequalities hold:

$$
0 \leq z(r)<z\left(r_{*}+0\right) ; \quad\left[R_{d}-r_{*}\right] \cdot \tan \alpha_{c} \leq z\left(r_{*}+0\right) \leq\left[R_{d}-r_{*}\right] \cdot \tan \left(\frac{\pi}{2}-\alpha_{g}\right) .
$$

We will show now that $r_{*} \geq R_{d} / n$ and $z^{\prime}\left(r_{*}+0\right)=-\tan \left(\pi / 2-\alpha_{g}\right)$.

In order to show that $r_{*} \geq R_{d} / n$ we assume the contrary, that is, $r_{*}<R_{d} / n$. Under this hypothesis we have

$$
\begin{aligned}
\alpha\left(\frac{R_{d}}{n}\right)-\alpha\left(R_{d}\right) & =-\alpha^{\prime}\left(r^{\prime}\right) \cdot\left(R_{d}-\frac{R_{d}}{n}\right) \\
= & \frac{1}{\cos \alpha\left(r^{\prime}\right)}\left[-\frac{p}{r}-\frac{\rho \cdot g \cdot z\left(r^{\prime}\right)}{r}+\frac{\sin \alpha\left(r^{\prime}\right)}{r^{\prime}}\right] \cdot\left(R_{d}-\frac{R_{d}}{n}\right) \\
> & \frac{R_{d}-R_{d} / n}{\cos \alpha\left(r^{\prime}\right)} \cdot\left[-\frac{\alpha_{c}+\alpha_{g}-\pi / 2}{R_{d}} \cdot \frac{n}{n-1} \cdot \cos \alpha_{c}+\frac{\rho \cdot g \cdot R_{d}}{r} \cdot \frac{n-1}{n} \cdot \tan \left(\frac{\pi}{2}-\alpha_{g}\right)\right. \\
& \left.-\frac{1}{R_{d}} \cdot \sin \alpha_{c}-\frac{\rho \cdot g \cdot z\left(r^{\prime}\right)}{r}+\frac{\sin \alpha\left(r^{\prime}\right)}{r^{\prime}}\right]>\frac{\pi}{2}-\left(\alpha_{c}+\alpha_{g}\right)
\end{aligned}
$$


for some $r^{\prime} \in\left(R_{d} / n, R_{d}\right)$. Hence $\alpha\left(R_{d} / n\right)>\pi / 2-\alpha_{g}$. This last inequality is impossible, according to the definition (3.21) of $r_{*}$. Therefore, $r_{*}$, defined by (3.21), satisfies $r_{*} \geq R_{d} / n$.

In order to show that $z^{\prime}\left(r_{*}+0\right)=-\tan \left(\pi / 2-\alpha_{g}\right)$, we remark that from the definition (3.21) of $r_{*}$ it follows that in $r_{*}$ at least one of the following three equalities hold:

$$
z^{\prime}\left(r_{*}+0\right)=-\tan \alpha_{c}, \quad z^{\prime \prime}\left(r_{*}+0\right)=0, \quad z^{\prime}\left(r_{*}+0\right)=-\tan \left(\frac{\pi}{2}-\alpha_{g}\right) .
$$

Since $z^{\prime}\left(r_{*}+0\right)<z^{\prime}(r) \leq-\tan \alpha_{c}$ for any $r \in\left(r_{*}, R_{d}\right]$, it follows that the equality $z^{\prime}\left(r_{*}+0\right)=$ $-\tan \alpha_{c}$ is impossible. Hence, we deduce that at $r_{*}$ at least one of the following two equalities hold: $z^{\prime \prime}\left(r_{*}+0\right)=0, z^{\prime}\left(r_{*}+0\right)=-\tan \left(\pi / 2-\alpha_{g}\right)$.

We show now that the equality $z^{\prime \prime}\left(r_{*}+0\right)=0$ is impossible. For that we assume the contrary, that is, $z^{\prime \prime}\left(r_{*}+0\right)=0$. Under this hypothesis, from (3.17) we have

$$
\begin{aligned}
p= & -\rho \cdot g \cdot z\left(r_{*}+0\right)+\frac{\gamma}{r_{*}} \cdot \sin \alpha\left(r_{*}+0\right) \geq-\rho \cdot g \cdot\left(R_{d}-r_{*}\right) \cdot \tan \left(\frac{\pi}{2}-\alpha_{g}\right)+\frac{\gamma}{r_{*}} \cdot \sin \alpha\left(r_{*}+0\right) \\
> & \frac{\gamma}{R_{d}} \cdot \sin \alpha_{c}-\rho \cdot g \cdot R_{d} \cdot \frac{n-1}{n} \cdot \tan \left(\frac{\pi}{2}-\alpha_{g}\right)>\frac{\gamma}{R_{d}} \cdot \sin \alpha_{c}-\rho \cdot g \cdot R_{d} \cdot \frac{n-1}{n} \cdot \tan \left(\frac{\pi}{2}-\alpha_{g}\right) \\
& +\gamma \cdot \frac{\alpha_{c}+\alpha_{g}-\pi / 2}{R_{d}} \cdot \frac{n}{n-1} \cdot \cos \alpha_{c}
\end{aligned}
$$

what is impossible.

In this way we obtain that the equality $z^{\prime}\left(r_{*}+0\right)=-\tan \left(\pi / 2-\alpha_{g}\right)$ holds.

Taking now $R_{c}=r_{*}$ we find that the solution of the initial value problem (3.17) on the interval $\left[R_{c}, R_{d}\right]$ is convex, and it is the meridian curve of an appropriate meniscus.

Corollary 3.14. If $\alpha_{c}<\pi / 2-\alpha_{g}$ and

$$
p<\gamma \cdot \frac{\alpha_{c}+\alpha_{g}-\pi / 2}{R_{d}} \cdot \cos \alpha_{c}-\rho \cdot g \cdot R_{d} \cdot \tan \left(\frac{\pi}{2}-\alpha_{g}\right),
$$

then there exists $R_{c} \in\left(0, R_{d}\right]$ such that the solution of the initial value problem (3.17) is a globally convex solution of the NLBVP (2.5) and (2.6) on $\left[R_{c}, R_{d}\right]$.

Corollary 3.15. If $\alpha_{c}<\pi / 2-\alpha_{g}, n>n^{\prime}>1$ and the inequalities hold

$$
\begin{aligned}
\gamma \cdot \frac{\alpha_{c}+\alpha_{g}-\pi / 2}{R_{d}} \cdot \frac{n^{\prime}}{n^{\prime}-1} \cdot \sin \alpha_{g}+\frac{\gamma}{R_{d}} \cdot n^{\prime} \cos \alpha_{g} \\
<p<\gamma \cdot \frac{\alpha_{c}+\alpha_{g}-\pi / 2}{R_{d}} \cdot \frac{n}{n-1} \cdot \cos \alpha_{c} \\
\quad-\rho \cdot g \cdot R_{d} \cdot \frac{n-1}{n} \cdot \tan \left(\frac{\pi}{2}-\alpha_{g}\right)+\frac{\gamma}{R_{d}} \cdot \sin \alpha_{c}
\end{aligned}
$$

then there exists $R_{c} \in\left[R_{d} / n, R_{d} / n^{\prime}\right]$ such that the solution of the initial value problem (3.17) is a globally convex solution of the NLBVP $(2.5),(2.6)$ on $\left[R_{c}, R_{d}\right]$. 
The existence of $R_{c}$ and the inequality $R_{c}>R_{d} / n$ follows from Theorem 3.13. The inequality $R_{c}<R_{d} / n^{\prime}$ follows from the Corollary 3.4 of Theorem 3.3.

Theorem 3.16 (sufficient condition for the existence of globally concave solution). Let $n>1$ be a real number. If $\alpha_{c}>\pi / 2-\alpha_{g}$ and $p$ satisfies

$$
p<\gamma \cdot \frac{\alpha_{c}+\alpha_{g}-\pi / 2}{R_{d}} \cdot \frac{n}{n-1} \cdot \sin \alpha_{g}+\frac{\gamma}{R_{d}} \cdot n \cdot \sin \alpha_{c}
$$

then there exists $R_{c}$ in the closed interval $\left[R_{d} / n, R_{d}\right]$ such that the solution of the initial value problem

$$
\begin{gathered}
z^{\prime \prime}=-\frac{\rho \cdot g \cdot z+p}{r}\left[1+\left(z^{\prime}\right)^{2}\right]^{3 / 2}-\frac{1}{r}\left[1+\left(z^{\prime}\right)^{2}\right] \cdot z^{\prime} \quad 0<r<R_{d}, R_{d}>0 \\
z\left(R_{d}\right)=0, \quad z^{\prime}\left(R_{d}\right)=-\tan \alpha_{c} \quad 0<\frac{\pi}{2}-\alpha_{g}<\alpha_{c}<\frac{\pi}{2}
\end{gathered}
$$

is a globally concave solution of the NLBVP (2.5) and (2.6) on $\left[R_{c}, R_{d}\right]$.

Proof. Consider the solution $z(r)$ of IVP (3.29). Denote by $I$ the maximal interval on which the function $z(r)$ exists and by $\alpha(r)$ the function $\alpha(r)=-\arctan z^{\prime}(r)$ defined on $I$. For the function $\alpha(r)$ the following equality holds:

$$
\alpha^{\prime}(r)=\frac{1}{\cos \alpha(r)} \cdot\left[\frac{p}{\gamma}+\frac{\rho \cdot g \cdot z(r)}{r}-\frac{\sin \alpha}{r}\right]
$$

Since

$$
\begin{gathered}
z^{\prime \prime}\left(R_{d}\right)=-\frac{\alpha^{\prime}\left(R_{d}\right)}{\cos ^{2} \alpha\left(R_{d}\right)}=-\frac{1}{\cos ^{3} \alpha\left(R_{d}\right)}\left[\frac{p}{\gamma}-\frac{\sin \alpha\left(R_{d}\right)}{R_{d}}\right]<0, \\
z^{\prime}\left(R_{d}\right)=-\tan \alpha_{c}<0, \\
z^{\prime}\left(R_{d}\right)<-\tan \left(\frac{\pi}{2}-\alpha_{g}\right),
\end{gathered}
$$

there exists $r^{\prime} \in I, 0<r^{\prime}<R_{d}$ such that for any $r \in\left[r^{\prime}, R_{d}\right]$ the following inequalities hold:

$$
z^{\prime \prime}(r)<0, \quad z^{\prime}(r) \geq-\tan \alpha_{c,} \quad z^{\prime}(r) \leq-\tan \left(\frac{\pi}{2}-\alpha_{g}\right) .
$$

Let now $r_{*}$ be defined by

$$
r_{*}=\inf \left\{r^{\prime} \in I \mid 0<r^{\prime}<R_{d} \text { such that for any } r \in\left[r^{\prime}, R_{d}\right] \text { inequalities (3.32) hold }\right\} .
$$


It is clear that $r_{*} \geq 0$ and for any $r \in\left(r_{*}, R_{d}\right]$ inequalities (3.32) hold. Moreover, $z^{\prime}\left(r_{*}+0\right)=$ $\lim _{r \rightarrow r_{*}, r>r_{*}} z^{\prime}(r)$ exists and satisfies $-\tan \alpha_{c} \leq z^{\prime}(r) \leq-\tan \left(\pi / 2-\alpha_{g}\right)$. Hence $z\left(r_{*}+0\right)=$ $\lim _{r \rightarrow r_{*}, r>r_{*}} z(r)$ is finite, it is strictly positive and for every $r \in\left(r_{*}, R_{d}\right]$ the inequalities hold:

$$
0 \leq z(r) \leq z\left(r_{*}+0\right) ; \quad\left[R_{d}-r_{*}\right] \cdot \tan \left(\frac{\pi}{2}-\alpha_{g}\right) \leq z\left(r_{*}+0\right) \leq\left[R_{d}-r_{*}\right] \cdot \tan \alpha_{c} .
$$

We will show now that $r_{*} \geq R_{d} / n$ and $z^{\prime}\left(r_{*}+0\right)=-\tan \left(\pi / 2-\alpha_{g}\right)$.

In order to show the inequality $r_{*} \geq R_{d} / n$ we assume the contrary, that is, that $r_{*}<$ $R_{d} / n$. Under this hypothesis we have

$$
\begin{aligned}
& \alpha\left(R_{d}\right)-\alpha\left(\frac{R_{d}}{n}\right)= \alpha^{\prime}\left(r^{\prime}\right) \cdot\left(R_{d}-\frac{R_{d}}{n}\right)=\frac{R_{d}-R_{d} / n}{\cos \alpha\left(r^{\prime}\right)}\left[\frac{p}{r}+\frac{\rho \cdot g \cdot z\left(r^{\prime}\right)}{r}-\frac{\sin \alpha\left(r^{\prime}\right)}{r^{\prime}}\right] \\
&>\frac{R_{d} \cdot((n-1) / n)}{\cos \alpha\left(r^{\prime}\right)} \cdot\left[\frac{\alpha_{c}+\alpha_{g}-\pi / 2}{R_{d}} \cdot \frac{n}{n-1} \cdot \sin \alpha_{g}\right. \\
&\left.+\frac{n}{R_{d}} \cdot \sin \alpha_{\mathrm{c}}+\frac{\rho \cdot g \cdot z\left(r^{\prime}\right)}{r}-\frac{\sin \alpha\left(r^{\prime}\right)}{r^{\prime}}\right]
\end{aligned}
$$

for some $r^{\prime} \in\left(R_{d} / n, R_{d}\right)$. Since $\left(n / R_{d}\right) \cdot \sin \alpha_{c}>\left(n / r^{\prime}\right) \cdot \sin \alpha\left(r^{\prime}\right), \sin \alpha_{g} / \cos \alpha\left(r^{\prime}\right)>1$, and $\rho \cdot g \cdot z\left(r^{\prime}\right) / \gamma>0$, it follows that $\alpha\left(R_{d}\right)-\alpha\left(R_{d} / n\right)>\alpha_{c}+\alpha_{g}-\pi / 2$. Hence $\alpha\left(R_{d} / n\right)>\pi / 2-\alpha_{g}$, what is impossible, according to the definition of $r_{*}$. Therefore, $r_{*}$, defined by (3.33), satisfies $r_{*} \geq R_{d} / n$.

In order to show that $z^{\prime}\left(r_{*}+0\right)=-\tan \left(\pi / 2-\alpha_{g}\right)$, we remark that from the definition (3.33) of $r_{*}$ it follows that in $r_{*}$ at least one of the following three equalities holds:

$$
z^{\prime}\left(r_{*}+0\right)=-\tan \alpha_{c}, \quad z^{\prime \prime}\left(r_{*}+0\right)=0, \quad z^{\prime}\left(r_{*}+0\right)=-\tan \left(\frac{\pi}{2}-\alpha_{g}\right) .
$$

Since $z^{\prime}\left(r_{*}+0\right)>z^{\prime}(r)>-\tan \alpha_{c}$ for any $r \in\left(r_{*}, R_{d}\right]$, it follows that the equality $z^{\prime}\left(r_{*}+0\right)=$ $-\tan \alpha_{c}$ is impossible. Hence, we deduce that at $r_{*}$ at least one of the following two equalities holds: $z^{\prime \prime}\left(r_{*}+0\right)=0, z^{\prime}\left(r_{*}+0\right)=-\tan \left(\pi / 2-\alpha_{g}\right)$.

We show now that the equality $z^{\prime \prime}\left(r_{*}+0\right)=0$ is impossible. For that we assume the contrary, that is, $z^{\prime \prime}\left(r_{*}+0\right)=0$. Under this hypothesis, from (3.29) we have $p=-\rho \cdot g \cdot z\left(r_{*}+\right.$ $0)+\left(\gamma / r_{*}\right) \cdot \sin \alpha\left(r_{*}+0\right)<\left(n \cdot \gamma / R_{d}\right) \cdot \sin \alpha_{c}$, what is impossible.

In this way we obtain that the equality $z^{\prime}\left(r_{*}+0\right)=-\tan \left(\pi / 2-\alpha_{g}\right)$ holds.

Taking now $R_{c}=r_{*}$ we find that the solution of the initial value problem (3.29) on the interval $\left[R_{c}, R_{d}\right]$ is concave, and it is the meridian curve of an appropriate meniscus. 
Corollary 3.17. If $\alpha_{c}>\pi / 2-\alpha_{g}, n>n^{\prime}>1$ and the inequalities hold:

$$
\begin{gathered}
\gamma \cdot \frac{\alpha_{c}+\alpha_{g}-\pi / 2}{R_{d}} \cdot \frac{n}{n-1} \cdot \sin \alpha_{g}+\frac{\gamma}{R_{d}} \cdot n \cdot \sin \alpha_{c} \\
<p<\gamma \cdot \frac{\alpha_{c}+\alpha_{g}-\pi / 2}{R_{d}} \cdot \frac{n^{\prime}}{n^{\prime}-1} \cdot \cos \alpha_{c} \\
\quad-\rho \cdot g \cdot R_{d} \cdot \frac{n^{\prime}-1}{n^{\prime}} \cdot \tan \alpha_{c}+\frac{\gamma}{R_{d}} \cdot \sin \alpha_{c}
\end{gathered}
$$

then there exists $R_{c} \in\left[R_{d} / n, R_{d} / n^{\prime}\right]$ such that the solution of the IVP (3.29) is a globally concave solution of the NBVP (2.5), (2.6) on $\left[R_{c}, R_{d}\right]$.

The existence of $R_{c}$ and the inequality $R_{d} / n<R_{c}$ follows from Theorem 3.16. The inequality $R_{c}<R_{d} / n$ follows from Corollary 3.7. and Theorem 3.6.

\section{Stability of the Solution}

Definition 4.1. A solution $z(r)$ of the NLBVP (2.5), (2.6) stable if $z(r)$ is a minimum for the energy functional (2.9).

Theorem 4.2. If for $\alpha_{c}<\pi / 2-\alpha_{g}$ the NLBVP (2.5), (2.6) has a globally convex solution $z(r)$ on $\left[R_{c}, R_{d}\right]$ and the ratio $n=R_{d} / R_{c}>1$ satisfies:

$$
\frac{n-1}{n^{1 / 2}}<\pi \cdot \frac{1}{R_{d}} \cdot \frac{\gamma^{1 / 2} \cdot \sin ^{3 / 2} \alpha_{g}}{\rho^{1 / 2} \cdot g^{1 / 2}}
$$

then the solution is stable.

Proof. Since (2.5) is the Euler equation for the energy functional (2.9), it is sufficient to prove that the Legendre and Jacobi conditions are satisfied in this case.

It is easy to see that in this case the Legendre condition reduces to the inequality:

$$
\frac{r \cdot \gamma}{\left[1+\left(z^{\prime}\right)^{2}\right]^{3 / 2}}>0
$$

and it is satisfied.

By computation we find that the Jacobi equation in this case is

$$
\frac{d}{d r}\left(\frac{r \cdot r}{\left[1+\left(z^{\prime}\right)^{2}\right]^{3 / 2}} \cdot \frac{d \eta}{d r}\right)+\rho \cdot g \cdot z \cdot \eta=0
$$


For the coefficients of (4.3) the following inequality holds:

$$
\frac{r \cdot \gamma}{\left[1+\left(z^{\prime}\right)^{2}\right]^{3 / 2}} \geq \frac{R_{d}}{n} \cdot \gamma \cdot \sin ^{3} \alpha_{g}, \quad \rho \cdot g \cdot z \leq \rho \cdot g \cdot R_{d}
$$

Hence,

$$
\frac{d}{d r}\left(\frac{R_{d}}{n} \cdot r \cdot \sin ^{3} \alpha_{g} \cdot \frac{d \eta}{d r}\right)+\rho \cdot g \cdot R_{d} \cdot \eta=0
$$

is a Sturm type upper bound [19] for (4.3).

An arbitrary solution of (4.5) is given by

$$
\eta(r)=A \cdot \sin (\omega \cdot r+\varphi),
$$

where $A$ and $\varphi$ are real constants and $\omega^{2}$ is given by

$$
\omega^{2}=\frac{\rho \cdot g \cdot n}{\gamma \cdot \sin ^{3} \alpha_{g}}
$$

Inequality (4.1) implies that the half period $\pi / \omega$ of $\eta(r)$ satisfies the inequality $\pi / \omega>$ $R_{d}-R_{c}$. Hence a nonzero solution given by (4.6) has at most one zero on the interval $\left[R_{c}, R_{d}\right]$. Since any nonzero solution of (4.5) vanishes at most once on the interval $\left[R_{c}, R_{d}\right]$, the solution of (4.3) which satisfies $\eta\left(R_{d}\right)=0, \eta^{\prime}\left(R_{d}\right)=1$ has only one zero on $\left[R_{c}, R_{d}\right]$. Hence the Jacobi condition is satisfied in this case.

Theorem 4.3. If for $\alpha_{c}<\pi / 2-\alpha_{g}$ the NLBVP (2.5) and (2.6) has a globally convex solution $z(r)$ on $\left[R_{c}, R_{d}\right]$, and the ratio $n=R_{d} / R_{c}>1$ satisfies

$$
\frac{n-1}{n^{1 / 2}}>2 \pi \cdot \frac{1}{R_{d}} \cdot \frac{\gamma^{1 / 2} \cdot \cos ^{3 / 2} \alpha_{c}}{\rho^{1 / 2} \cdot g^{1 / 2}},
$$

then the meniscus is unstable.

Proof. We remark now that for the Jacobi equation the following inequalities hold:

$$
\frac{r \cdot r}{\left[1+\left(z^{\prime}\right)^{2}\right]^{3 / 2}} \leq R_{d} \cdot \gamma \cdot \cos ^{3} \alpha_{c}, \quad \rho \cdot g \cdot z \geq \rho \cdot g \cdot \frac{R_{d}}{n} \text {. }
$$

Hence

$$
\frac{d}{d r}\left[R_{d} \gamma \cdot \cos ^{3} \alpha_{c} \cdot \frac{d \eta}{d r}\right]+\rho \cdot g \cdot \frac{R_{d}}{n} \cdot \eta=0
$$

is a Sturm type lower bound [19] of the Jacobi equation (4.3). 
An arbitrary solution of (4.10) is given by

$$
\eta(r)=A \cdot \sin (\omega \cdot r+\varphi)
$$

where $\omega^{2}$ is given by

$$
\omega^{2}=\frac{\rho \cdot g}{n \cdot \gamma \cdot \cos ^{3} \alpha_{c}}
$$

Inequality (4.8) implies that $\eta(r)$ given by (4.11) vanishes twice on $\left[R_{c}, R_{d}\right]$. Therefore the Jacobi condition is not satisfied.

Theorem 4.4. If for $\pi / 2-\alpha_{g}<\alpha_{c}<\pi / 2$ the NLBVP (2.5), (2.6) has a globally-concave solution $z(r)$ on $\left[R_{c}, R_{d}\right]$ and the ratio $n=R_{d} / R_{c}>1$ satisfies:

$$
\frac{n-1}{n^{1 / 2}}<\pi \cdot \frac{1}{R_{d}} \cdot \frac{r^{1 / 2} \cdot \cos ^{3 / 2} \alpha_{c}}{\rho^{1 / 2} \cdot g^{1 / 2}}
$$

then the solution is stable.

Proof. In order to verify the Jacobi condition in this case, we remark that for the coefficients of the Jacobi equation (4.3) the following inequalities hold:

$$
\frac{r \cdot \gamma}{\left[1+\left(z^{\prime}\right)^{2}\right]^{3 / 2}} \geq \frac{R_{d}}{n} \cdot \gamma \cdot \cos ^{3} \alpha_{c}, \quad \rho \cdot g \cdot z \leq \rho \cdot g \cdot R_{d}
$$

Hence

$$
\frac{d}{d r}\left[\frac{R_{d}}{n} \cdot r \cdot \cos ^{3} \alpha_{c} \cdot \frac{d \eta}{d r}\right]+\rho \cdot g \cdot R_{d} \cdot \eta=0
$$

is a Sturm type upper bound [19] of the Jacobi equation (4.3).

An arbitrary solution of (4.15) is given by

$$
\eta(r)=A \cdot \sin (\omega \cdot r+\varphi),
$$

where $A$ and $\varphi$ are real constants and $\omega^{2}$ is given by

$$
\omega^{2}=\frac{\rho \cdot g \cdot n}{\gamma \cdot \cos ^{3} \alpha_{c}}
$$

Inequality (4.13) implies that the half period $\pi / \omega$ of $\eta(r)$ satisfies the inequality $\pi / \omega>R_{d}-$ $R_{c}$. It follows that a nonzero solution given by (4.16) has at most one zero on the interval $\left[R_{c}, R_{d}\right]$. Since any nonzero solution of (4.15) vanishes at most once on the interval $\left[R_{c}, R_{d}\right]$, the solution of the Jacobi equation (4.3), which satisfies $\eta\left(R_{d}\right)=0, \eta^{\prime}\left(R_{d}\right)=1$, has only one zero on $\left[R_{c}, R_{d}\right]$. Hence the Jacobi condition is satisfied. 
Theorem 4.5. If for $\pi / 2-\alpha_{g}<\alpha_{c}<\pi / 2$ the NLBVP (2.5) and (2.6) has a globally concave solution $z(r)$ on $\left[R_{c}, R_{d}\right]$ and the ratio $n=R_{d} / R_{c}>1$ satisfies

$$
\frac{n-1}{n^{3 / 2}}>2 \pi \cdot \frac{1}{R_{d}} \cdot \frac{\gamma^{1 / 2} \cdot \sin ^{3 / 2} \alpha_{g}}{\rho^{1 / 2} \cdot g^{1 / 2}}
$$

then the solution is unstable.

Proof. Remark that in this case for the coefficients of the Jacobi equation (4.3) the following inequalities hold:

$$
\frac{r \cdot \gamma}{\left[1+\left(z^{\prime}\right)^{2}\right]^{3 / 2}} \leq R_{d} \cdot \gamma \cdot \sin ^{3} \alpha_{g}, \quad \rho \cdot g \cdot z \geq \rho \cdot g \cdot \frac{R_{d}}{n} .
$$

From this step the proof is similar to the proof of Theorem 4.3.

\section{Numerical Illustration}

Numerical computations were performed for NdYAG microfiber growth by pulling down method using the following numerical data. $R_{d}=5 \times 10^{-4}[\mathrm{~m}] ; R_{c}=4 \times 10^{-4}[\mathrm{~m}] ; \alpha_{c}^{1}=$ $0.523[\mathrm{rad}] ; \alpha_{c}^{2}=1.4[\mathrm{rad}] ; \alpha_{g}=0.2967[\mathrm{rad}] ; \rho=3.6 \times 10^{3}\left[\mathrm{~kg} / \mathrm{m}^{3}\right] ; \gamma=7.81 \times 10^{-1}[\mathrm{~N} / \mathrm{m}] ;$ $g=9.81\left[\mathrm{~m} / \mathrm{s}^{2}\right]$.

The objective was to find the ranges for the values of $p$ (determined in Section 3) and to find $p$ for which meniscus, having prior given shape and size, exists.

(a) Inequality (3.2) establishes the range where $p$ has to be when $\alpha_{c}<\pi / 2-\alpha_{g}$ and a globally convex solution of the NLBVP (2.5) and (2.6) on $\left[R_{c}, R_{d}\right]$, exists. Computation shows that for the considered numerical data $\left(\alpha_{c}=\alpha_{c}^{1}\right)$ this range is $-4306.62 \mathrm{~Pa} \leq p \leq-219.94 \mathrm{~Pa}$.

To identify the $p$ value for which a globally convex solution of the NLBVP (2.5) and (2.6) exists on $\left[R_{c}, R_{d}\right]$, the IVP (5.1)

$$
\begin{aligned}
\frac{d z}{d t} & =-\tan \alpha \\
\frac{d \alpha}{d t} & =\frac{\rho \cdot g \cdot z+p}{r} \cdot \frac{1}{\cos \alpha}-\frac{1}{r} \cdot \tan \alpha \\
z\left(R_{d}\right) & =0, \quad \alpha\left(R_{d}\right)=\alpha_{c}
\end{aligned}
$$

has to be integrated numerically for $\alpha_{c}=\alpha_{c}^{1}$ and different values of $p$ in the above range. The dependence $r_{c}(p)$ has to be found, where $r_{c}(p)$ is defined by $\alpha\left(r_{c}(p)\right)=\pi / 2-\alpha_{g}$. The value of $p$ for which $r_{c}(p)$ is equal to $R_{c}$ is found solving the equation $r_{c}(p)=R_{c}$. If the function $r_{c}(p)$ does not exists (i.e., the growth angle is not reached) or the equation $r_{c}(p)=R_{c}$ has no solution, then there is no $p$ for which a globally convex solution of the NLBVP (2.5) and (2.6) on $\left[R_{c}, R_{d}\right]$ exists. For the considered numerical data the graphic of the function $r_{c}(p)$ is represented in Figure 2(a). This figure shows that for $p=-2300 \mathrm{~Pa}$ the equality $r_{c}(p)=R_{c}$ holds; that is, meniscus with prior given shape and size exists. The meridian curve at this meniscus is represented in Figure 2(b). 


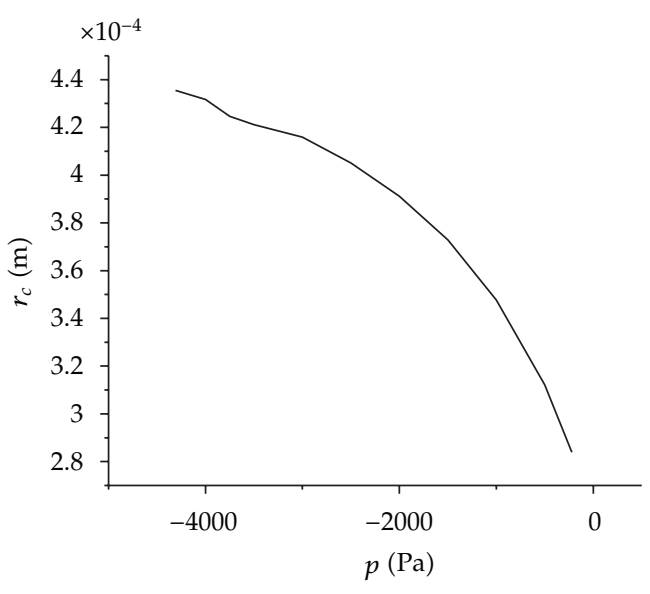

(a)

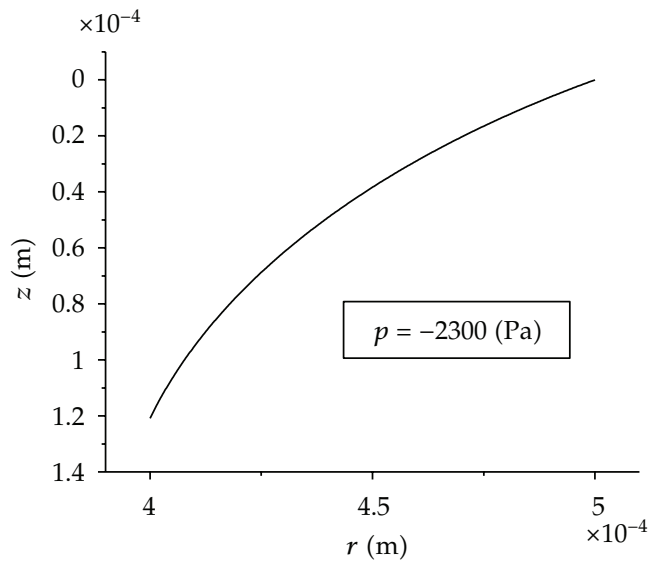

(b)

Figure 2: (a) The function $r_{c}(p)$ for $\alpha_{c}=\alpha_{c}^{1}=0.523 \mathrm{rad}$. (b) The convex meridian curve for $p=-2300 \mathrm{~Pa}$, suitable to grow microfiber with $r_{c}(p)=R_{c}=4 \times 10^{-4} \mathrm{~m}$.

(b) Inequality (3.9) establishes the range where $p$ has to be when $\alpha_{c}>\pi / 2-\alpha_{g}$ and a globally-concave solution of the NLBVP (2.5) and (2.6) on $\left[R_{c}, R_{d}\right]$ exists. Computation shows, that for the considered numerical data, $\alpha_{c} \alpha_{c}^{2}$, this range is $[1640,2211]$ Pa. To identify the value of $p$ the IVP (5.1) for $\alpha_{c}=\alpha_{c}^{2}$ and for different values of $p$ in the above range has to be solved. The dependence $r_{c}(p)$ has to be found, where $r_{c}(p)$ is defined by $\alpha\left(r_{c}(p)\right)=$ $\pi / 2-\alpha_{g}$. The value of $p$ is found solving the equation $r_{c}(p)=R_{c}$. If the function $r_{c}(p)$ does not exists (i.e., the growth angle is not reached) or the equation $r_{c}(p)=R_{c}$ has no solution, then there is no $p$ for which meniscus, having the prescribed shape and size, exists. For the considered numerical data the graphic of the function $r_{c}(p)$ is represented in Figure 3(a). This figure shows that for $p=1906.5 \mathrm{~Pa}$ the equality $r_{c}(p)=4 \times 10^{-4} \mathrm{~m}$ holds; that is, meniscus with prescribed shape and size exists. The meridian curve of this meniscus is represented in Figure 3(b).

(c) Inequality (3.13) establishes the range where $p$ has to be when $\alpha_{c}<\pi / 2-\alpha_{g}$ and the NLBVP (2.5) and (2.6) has a convex-concave solution on $\left[R_{c}, R_{d}\right]$. Computation shows, that for the considered numerical data, $\alpha_{c}=\alpha_{c}^{1}$, this range is [781, 1867] Pa. The value of $p$ can be identified integrating numerically the IVP (5.1) for $\alpha_{c}=\alpha_{c}^{1}$ and for different values of $p$ in the above range. The dependence $r_{c}(p)$ has to be found, where $r_{c}(p)$ is defined by $\alpha\left(r_{c}(p)\right)=\pi / 2-\alpha_{g}$. The value of $p$ is found solving the equation $r_{c}(p)=R_{c}$. If the function $r_{c}(p)$ does not exist (i.e., the growth angle is not reached) or the equation $r_{c}(p)=R_{c}$ has no solution, then there is no $p$ for which a meniscus, with the prior given shape and size on $\left[R_{c}, R_{d}\right]$ exists. For the considered numerical data the graphic of the function $r_{c}(p)$ is represented on Figure 4(a). This figure shows that equation $r_{c}(p)=4 \times 10^{-4} \mathrm{~m}$ has no solution. Therefore, a meniscus with the prior given shape and size does not exist in this case. A convex-concave meniscus obtained for $p=1000 \mathrm{~Pa}$ is presented on Figure 4(b), but this is inappropriate because $r_{c}(p) \neq R_{c}=4 \times 10^{-4} \mathrm{~m}$.

(d) Inequality (3.15) establishes the range where $p$ has to be when $\alpha_{c}>\pi / 2-\alpha_{g}$ and the NLBVP (2.5) and (2.6) has a concave-convex solution on $\left[R_{c}, R_{d}\right]$. Computation shows that for the considered numerical data, $\alpha_{c}=\alpha_{c}^{2}$, this range is [1493.72, 1539.2] Pa. The value of $p$ can be identified integrating numerically the IVP (5.1) for $\alpha_{c}=\alpha_{c}^{2}$ and for different values 


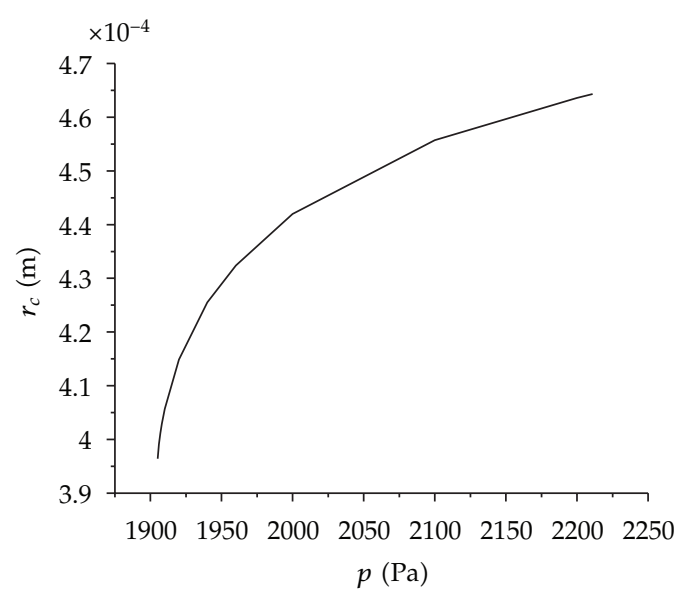

(a)

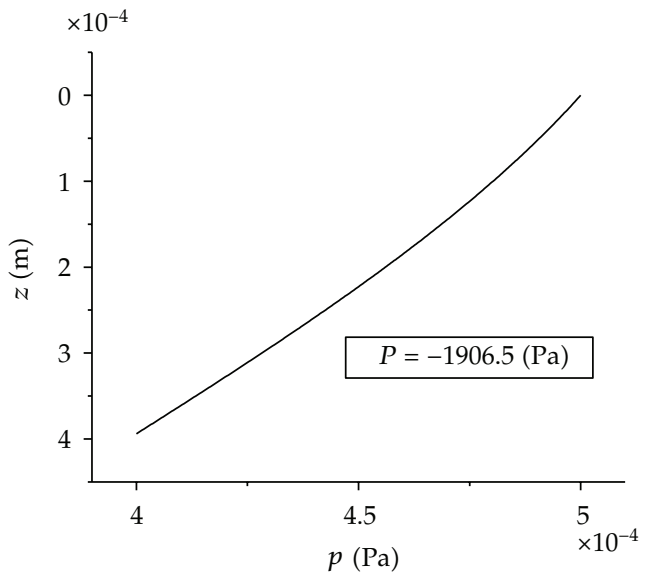

(b)

Figure 3: (a) The function $r_{c}(p)$ for $\alpha_{c}=\alpha_{c}^{2}=1.4 \mathrm{rad}$. (b) The concave meridian curve for $p=1906.5 \mathrm{~Pa}$, suitable to grow microfiber with $r_{c}(p)=R_{c}=4 \times 10^{-4} \mathrm{~m}$.

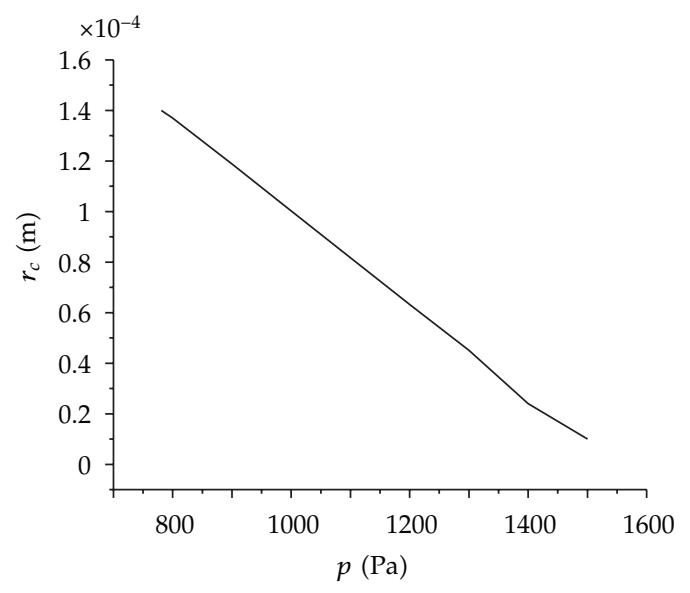

(a)

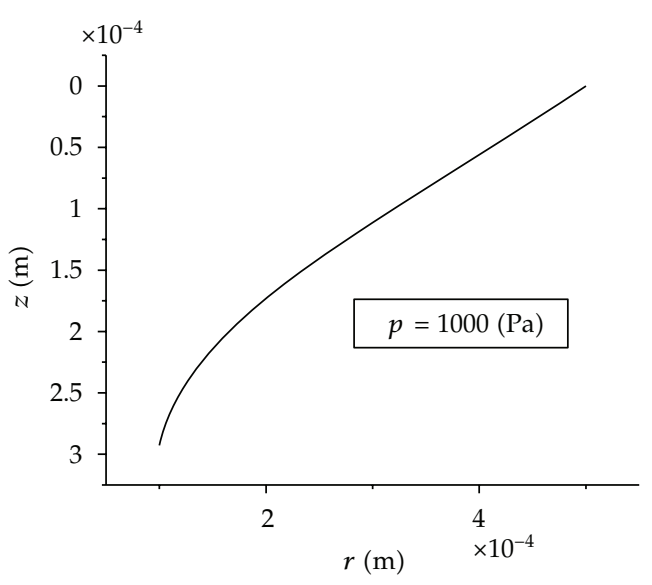

(b)

Figure 4: (a) The function $r_{c}(p)$ for $\alpha_{c}=\alpha_{c}^{1}=0.523 \mathrm{rad}$. (b) The convex-concave meridian curve for $p=$ $1000 \mathrm{~Pa}$, suitable to grow microfiber with $1 \times 10^{-4} \mathrm{~m}$.

of $p$ in the above range. The dependence $r_{c}(p)$ has to be found, where $r_{c}(p)$ is defined by $\alpha\left(r_{c}(p)\right)=\pi / 2-\alpha_{g}$. The value of $p$ is found solving the equation $r_{c}(p)=R_{c}$. If the function $r_{c}(p)$ does not exist (i.e., the growth angle is not reached), or the equation $r_{c}(p)=R_{c}$ has no solution, then there is no $p$ for which a meniscus having the prior given shape and size exists on $\left[R_{c}, R_{d}\right]$. For the considered numerical data computation shows that for $p$ in the range [1493.72, 1539.2] Pa the growth angle on the meridian curve (i.e., the solution of IVP (5.1) for $\alpha_{c}=\alpha_{c}^{2}$ ) is not reached. Therefore, the function $r_{c}(p)$ does not exist, and there is no concave-convex meniscus with the prescribed shape and size in this case.

(e) Inequality (3.15) establishes as well the range where $p$ has to be when $\alpha_{c}<\pi / 2-$ $\alpha_{g}$ and the NLBVP (2.5) and (2.6) has a concave-convex solution on $\left[R_{c}, R_{d}\right]$. Computation 


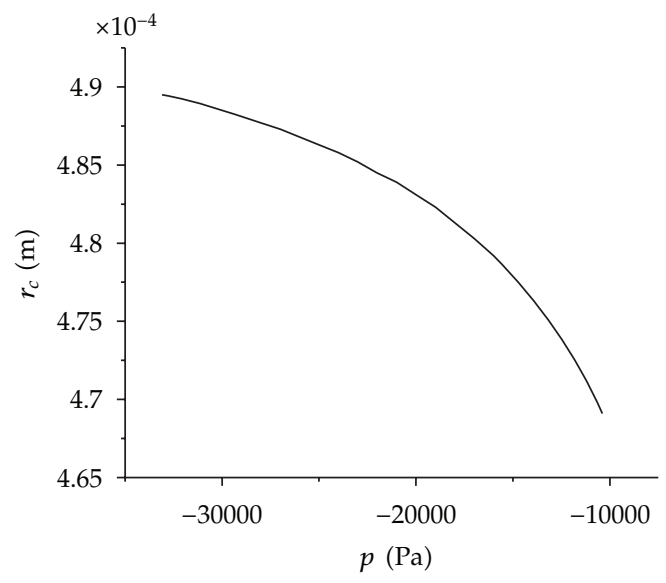

(a)

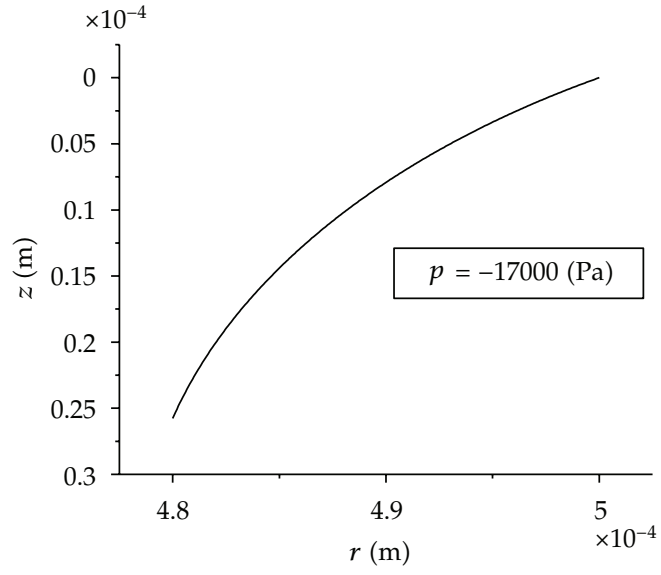

(b)

Figure 5: (a) The function $r_{c}(p)$ for $\alpha_{c}=\alpha_{c}^{1}=0.523 \mathrm{rad}, n=1.1, n^{\prime}=1.01$. (b) The convex meridian curve for $p=-17000 \mathrm{~Pa}$, suitable to grow microfiber with radius $4.8 \times 10^{-4} \mathrm{~m}$.

shows, that for the considered numerical data, $\alpha_{c}=\alpha_{c}^{1}$, this range is [1493.72, 1539.2] Pa. For $p$ in this range the meniscus shape is convex-concave, as it was shown in case (c). Therefore, for the considered data there is no meniscus with concave-convex shape.

(f) Inequality (3.13) establishes as well the range where $p$ has to be when $\alpha_{c}>\pi / 2-\alpha_{g}$ and the NLBVP (2.5), (2.6) has a convex-concave solution on $\left[R_{c}, R_{d}\right]$. Computation shows, that for the considered numerical data, $\alpha_{c}=\alpha_{c}^{1}$, this range is: [1539.2, 1867.18] Pa. The value of $p$ can be identified integrating numerically the IVP (5.1) for $\alpha_{c}=\alpha_{c}^{1}$ and for different values of $p$ in the above range. Following the method described at (c), computation shows that in this case the function $r_{c}(p)$ does not exist.

(g) Inequality (3.21) defines a range such that when $\alpha_{c}<\pi / 2-\alpha_{g}$ and $p$ is in the range, then there exists $r_{c}$ in the range $\left[R_{d} / n, R_{d} / n^{\prime}\right]$ and a globally convex solution of the NLBVP (2.5) and (2.6) on $\left[r_{c}, R_{d}\right]$. For the considered numerical data, $\alpha_{c}=\alpha_{c}^{1}, n=1.1>1.01=n^{\prime}>$ 1 , the range for $p$ is [-33110, -10390] Pa. Integrating numerically the IVP (5.1) for $\alpha_{c}=\alpha_{c}^{1}$ and for different values of $p$ in this range, we will find $r_{c}$ in the range $\left[R_{d} / 1.1, R_{d} / 1.01\right]=$ $[4.54 ; 4.95] \times 10^{-4} \mathrm{~m}$ and a globally convex solution of the NLBVP $(2.5)$ and $(2.6)$ on $\left[r_{c}, R_{d}\right]$. For the considered numerical data the dependence $r_{c}(p)$ is represented in Figure 5(a) and the globally convex meniscus shape obtained for $p=-17000 \mathrm{~Pa}$ is presented in Figure 5(b).

(h) Inequality (3.37) defines a range such that when $\alpha_{c}>\pi / 2-\alpha_{g}$ and $p$ is in the range, then there exists $r_{c}$ in the range $\left[R_{d} / n, R_{d} / n^{\prime}\right]$ and a globally-concave solution of the NLBVP (2.5) and (2.6) on $\left[r_{c}, R_{d}\right]$. For the considered numerical data, $\alpha_{c}=\alpha_{c}^{2}, n=1.1>1.01=$ $n^{\prime}>1$, the range for $p$ is $[1836,2326] \mathrm{Pa}$. Integrating numerically the IVP (5.1) for $\alpha_{c}=\alpha_{c}^{2}$ and for different values of $p$ in this range, we will find $r_{c}$ in the range $\left[R_{d} / 1.1, R_{d} / 1.01\right]=$ $[4.54 ; 4.95] \times 10^{-4} \mathrm{~m}$ and a globally-concave solution of the $\operatorname{NLBVP}(2.5)$ and $(2.6)$ on $\left[r_{c}, R_{d}\right]$. For the considered numerical data the dependence $r_{c}(p)$ is represented in Figure 6(a) with continuous line. It turns out that for the same $\alpha_{c}=\alpha_{c}^{2}$ and $p$ in the mentioned range we find globally-concave and a convex-concave solution of the NLBVP (2.5) and (2.6). For the convex-concave solution $r_{c}$ is less than $R_{d} / 1.1=4.54 \times 10^{-4} \mathrm{~m}$. The radius of these fibers is presented in Figure 6(a) with dot line. In Figure 6(b) such a convex-concave meniscus is presented, obtainable for $p=2200 \mathrm{~Pa}$. What happens in fact is that the growth angle is reached 


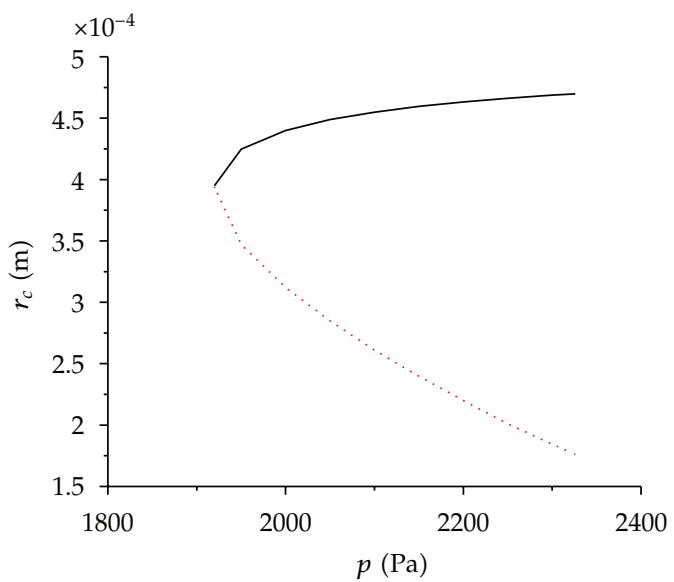

(a)

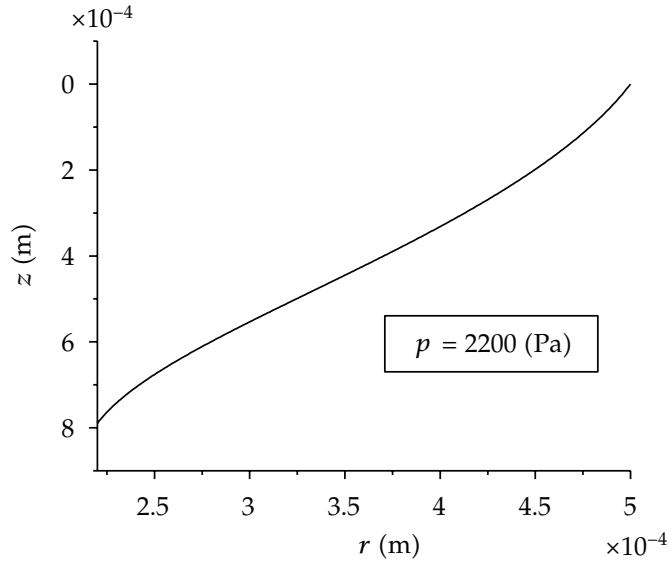

(b)

Figure 6: (a) The function $r_{c}(p)$ for $\alpha_{c}=\alpha_{c}^{2}=1.4 \mathrm{rad}, n=1.1, n^{\prime}=1.01$; (b) The convex-concave meridian curve for $p=2200 \mathrm{~Pa}$, suitable to grow microfiber with radius $2.2 \times 10^{-4} \mathrm{~m}$.

at $r_{c}^{1}=4.632 \times 10^{-4} \mathrm{~m}$ and $r_{c}^{2}=2.2 \times 10^{-4} \mathrm{~m}$, respectively. If the thermal field is fixed such that the solidification conditions are satisfied at the level $z=h_{c}^{1}=1.68 \times 10^{-4} \mathrm{~m}$, then the fiber radius will be $r_{c}^{1}=4.632 \times 10^{-4} \mathrm{~m}$. When the thermal field is fixed such that the solidification conditions are satisfied at the level $z=h_{c}^{2}=7 \times 10^{-4} \mathrm{~m}$, then the fiber radius will be $r_{c}^{2}=$ $2.2 \times 10^{-4} \mathrm{~m}$.

\section{Conclusion}

The above results are necessary or sufficient conditions for the existence and stability of a nonlinear boundary value problem occurring in microfiber crystal growth by pulling-down method. They can be used for the evaluation of the shape and size of the meniscus.

\section{Acknowledgment}

This work was supported by a grant of the Romanian National Authority for Scientific Research, CNCS-UEFISCDI, project no. PN-II-ID-PCE-2011-3-0171.

\section{References}

[1] L. Hesselink and S. Redfield, "Photorefractive holographic recording in strontium barium niobate fibers," Optics Letters, vol. 13, no. 10, pp. 877-879, 1988.

[2] S. Sudo, I. Yokohama, A. Cordova-Plazza, M. M. Fejer, and R. L. Ryer, "Uniform refractive index cladding for $\mathrm{LiNbO}_{3}$ single-crystal fibers," Applied Physics Letters, vol. 56, no. 20, pp. 1931-1938, 1990.

[3] H. Yoshinaga and K. Kitayama, "Holographic image storage in iron-doped lithium niobate fibers," Applied Physics Letters, vol. 56, no. 18, pp. 1728-1730, 1990.

[4] R. S. Feigelson, "Growth of fiber crystals," in Crystal Growth of Electronic Materials, E. Kaldis, Ed., p. 127, North-Holland, Amsterdam, The Netherland, 1985.

[5] D. H. Yoon, I. Yonenaga, T. Fukuda, and N. Ohnishi, "Crystal growth of dislocation-free $\mathrm{LiNbO}_{3}$ single crystals by micro pulling down method," Journal of Crystal Growth, vol. 142, no. 3-4, pp. 339343, 1994. 
[6] Y. M. Yu, V. I. Chani, K. Shimamura, K. Inaba, and T. Fukuda, "Growth of vanadium garnet fiber crystals and variations of lattice parameter," Journal of Crystal Growth, vol. 177, no. 1-2, pp. 74-78, 1997.

[7] B. M. Epelbaum, K. Imaba, S. Uda, and T. Fukuda, "A double-die modification of micro-pulling-down method for in situ clad/core doping of fiber crystal," Journal of Crystal Growth, vol. 179, no. 3-4, pp. 559-566, 1997.

[8] K. Shimamma, S. Uda, T. Yamada, S. Sakaguchi, and T. Fukuda, "Photoconductive behavior in smectic A phase of 2-(4'-heptyloxyphenyl)-6-dodecylthiobenzothiazole," Japanese Journal of Applied Physics, vol. 35, pp. L703-L705, 1996.

[9] N. Schäfer, T. Yamada, K. Shimamura, H. J. Koh, and T. Fukuda, "Growth of $\mathrm{Si}_{x} \mathrm{Ge}_{1-x}$ crystals by the micro-pulling-down method," Journal of Crystal Growth, vol. 166, no. 1-4, pp. 675-679, 1996.

[10] C. W. Lan, S. Uda, and T. Fukuda, "Theoretical analysis of the micro-pulling-down process for $\mathrm{Ge}_{x} \mathrm{Si}_{1-x}$ fiber crystal growth," Journal of Crystal Growth, vol. 193, no. 4, pp. 552-562, 1998.

[11] B. M. Epelbaum, K. Shimamura, S. Uda, J. Kon, and T. Fukuda, “Operating limits for stable growth of silicon fibers with diameter less than $150 \mu \mathrm{m}$ by modified $\mu$-PD method," Crystal Research and Technology, vol. 31, no. 8, pp. 1077-1084, 1996.

[12] H. J. Koh, N. Schäfer, K. Shimamura, and T. Fukuda, " $\mathrm{Si}_{1-x} \mathrm{Ge}_{x}$ fiber crystals with functionally variant composition grown by micro-pulling-down technique," Journal of Crystal Growth, vol. 167, no. 1-2, pp. 38-44, 1996.

[13] S. Uda, J. Kon, K. Shimamura, and T. Fukuda, “Analysis of Ge distribution in $\mathrm{Si}_{1-x} \mathrm{Ge}_{x}$ single crystal fibers by the micro-pulling down method," Journal of Crystal Growth, vol. 167, no. 1-2, pp. 64-73, 1996.

[14] C. J. Chang and R. A. Brown, "Radial segregation induced by natural convection and melt/solid interface shape in vertical bridgman growth," Journal of Crystal Growth, vol. 63, no. 2, pp. 343-364, 1983.

[15] P. M. Adornato and R. A. Brown, "Convection and segregation in directional solidification of dilute and non-dilute binary alloys: effects of ampoule and furnace design," Journal of Crystal Growth, vol. 80, no. 1, pp. 155-190, 1987.

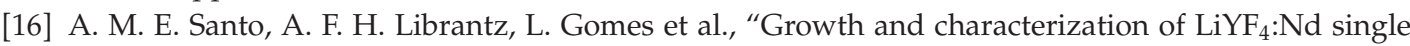
crystal fibres for optical applications," Journal of Crystal Growth, vol. 292, no. 1, pp. 149-154, 2006.

[17] R. Finn, Equilibrium Capillary Surfaces, vol. 284 of Grundlehren der mathematischen Wissenschaften, Springer, New York, NY, USA, 1986.

[18] V. A. Tatarchenko, Shaped Crystal Growth, Kluwer Academic, Dordrecht, The Netherlands, 1993.

[19] P. Hartman, Ordinary Differential Equations, John Wiley \& Sons, New York, NY, USA, 1964. 


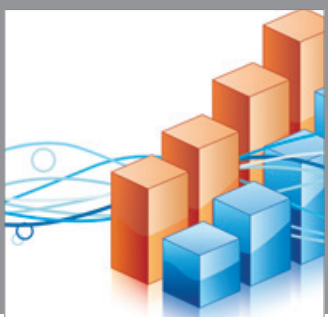

Advances in

Operations Research

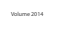

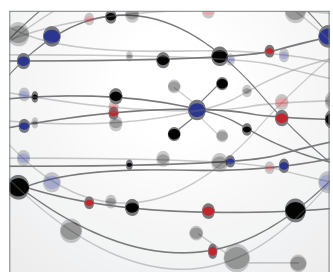

\section{The Scientific} World Journal
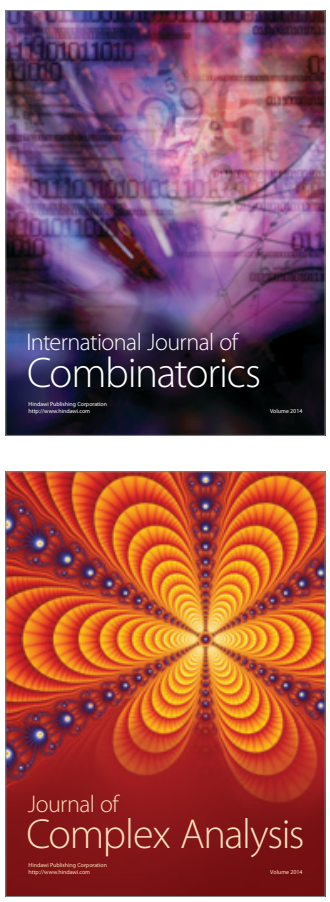

International Journal of

Mathematics and

Mathematical

Sciences
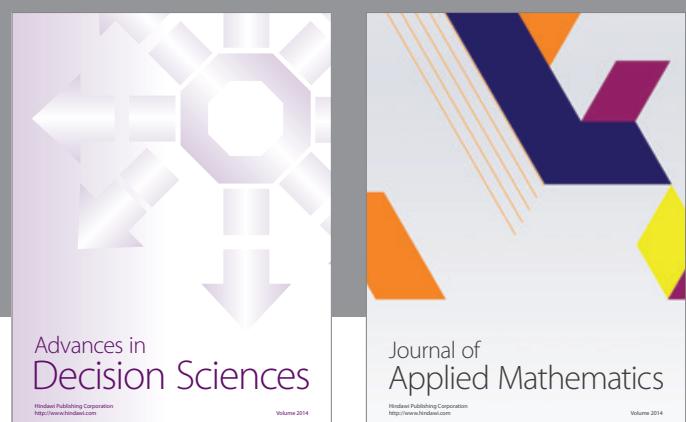

Journal of

Applied Mathematics
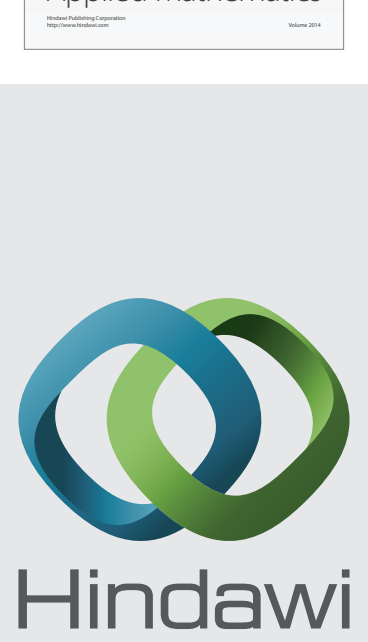

Submit your manuscripts at http://www.hindawi.com
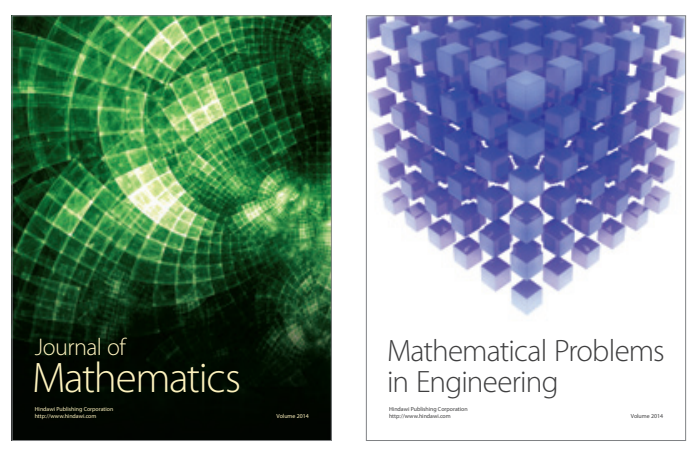

Mathematical Problems in Engineering
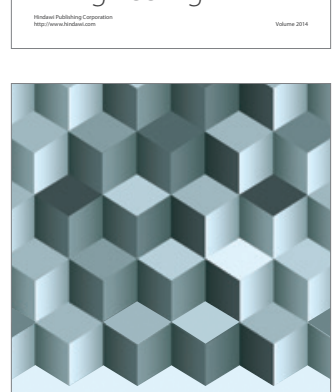

Journal of

Function Spaces
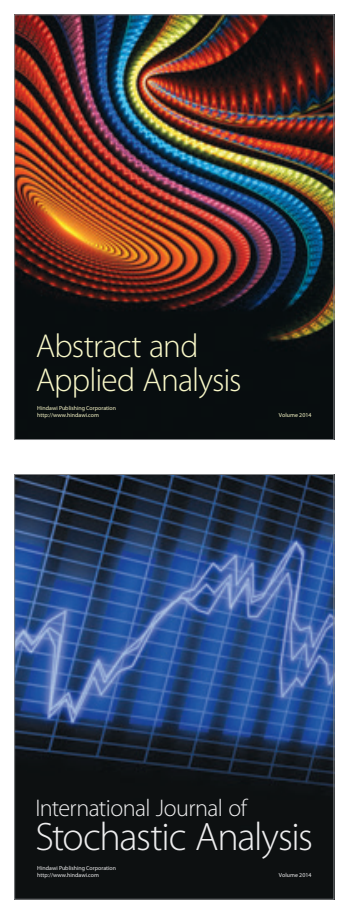

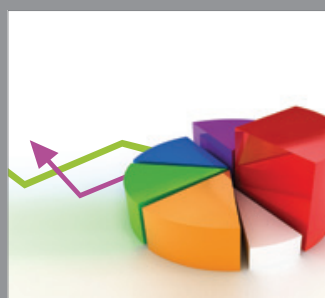

ournal of

Probability and Statistics

Promensencen
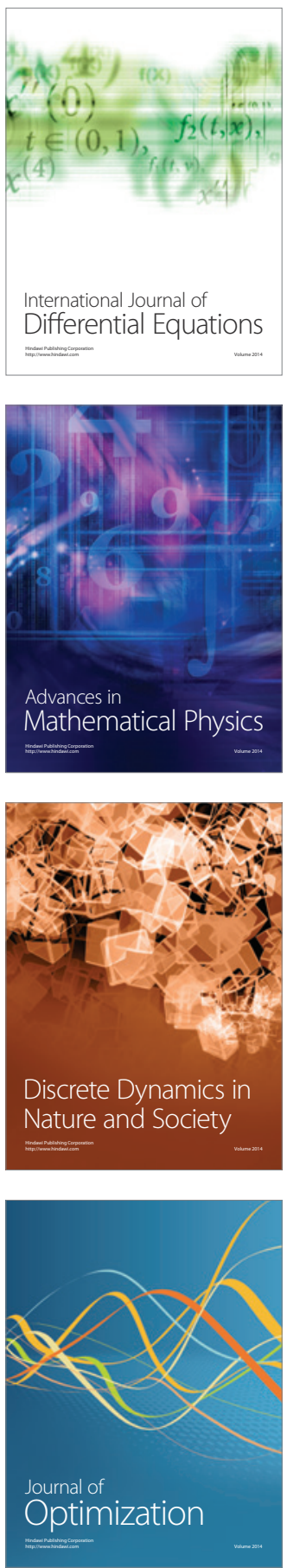